\title{
Role of Parietal Epithelial Cells in Kidney Injury: The Case of Rapidly Progressing Glomerulonephritis and Focal and Segmental Glomerulosclerosis
}

\author{
Marcus J. Moeller Bart Smeets \\ Nephrology and Clinical Immunology, Medizinische Klinik II, University Hospital of the RWTH Aachen \\ University, Aachen, Germany
}

\section{Key Words \\ Parietal epithelial cell · Glomerulonephritis · Podocyte · \\ Crescent $\cdot$ Glomerulosclerosis}

\begin{abstract}
Background: Millions of people are affected by irreversible loss of renal function and thus by a significantly increased cardiovascular risk. In this context, the parietal epithelial cells (PECs) of the glomerulus have attracted increasing attention in recent years. So far, they have been ascribed 2 major functions: (1) PECs may act as intrinsic progenitor cells to replenish podocytes and/or proximal tubular cells and (2) a major role of PECs has been proposed in 2 glomerular disease entities [i.e. rapidly progressing glomerulonephritis (RPGN) and focal and segmental glomerulosclerosis (FSGS)]. Summary: In this review, the major recent findings regarding the role of PECs in glomerular disease are summarized. Novel transgenic technologies have allowed major advances, in particular cell fate-tracing studies. Key Messages: Using these methods, it could be established that the proliferating cells in Bowman's space, which are characteristically found in RPGN, are derived almost exclusively from the glomerular epithelium - primarily PECs. Similarly, it could be shown that PECs participate in the formation of sclerotic le-
\end{abstract}

sions in FSGS. Since PECs deposit their characteristic extracellular matrix within these lesions, they likely contribute to the sclerotic process. A common feature of both diseases is that PECs are 'activated', i.e. PECs acquire a larger cytoplasm and nucleus and show increased migration and/or proliferation. Activated PECs can be identified by de novo expression of the marker CD44. These findings broaden our understanding of the pathogenesis of 2 different glomerular diseases: RPGN and FSGS. The participation of activated PECs in both diseases identifies these cells as prime pharmacological targets to develop more specific therapies for both diseases.

(c) 2014 S. Karger AG, Basel

\section{Introduction}

Only in recent years have the parietal epithelial cells (PECs) of the glomerulus attracted a significant amount of interest. This 'late' discovery is no surprise since PECs are probably the most inconspicuous cells in the periphery of the glomerulus. So far, it has been assumed that PECs function simply as 'wall paper' on the inner aspect of Bowman's capsule. However, more recently several potentially highly relevant and unexpected functions of PECs have been proposed. The most notable functions

\section{KARGER}

E-Mail karger@karger.com

www.karger.com/nee
(C) 2014 S. Karger AG, Basel

$1660-2129 / 14 / 1262-0097 \$ 39.50 / 0$
Marcus J. Moeller

Medizinische Klinik II, University Hospital of the RWTH Aachen University Pauwelsstrasse 30

DE-52074 Aachen (Germany)

E-Mail mmoeller@ukaachen.de 
are: (1) that PECs may serve as an intrinsic fixed progenitor population to replenish podocytes or proximal tubular cells and/or (2) that PECs may be involved in the pathogenesis of several glomerular diseases. While there is an ongoing debate regarding the first hypothesis, this review will focus on the second hypothesis, where the experimental data is more consistent and a consensus of the research community appears much more imminent.

\section{Activation of PECs: A Central Common Finding in Glomerular Disease}

PECs may show 2 very different phenotypes: under physiological conditions, PECs are very flat cells with a flat condensed nucleus and they show very little proliferative activity. PECs can become activated in 2 seemingly very different glomerular diseases: rapidly progressing glomerulonephritis (RPGN) and focal and segmental glomerulosclerosis (FSGS). Similar to other 'activated' cell populations, activated PECs show a very different morphology: they form a larger cytoplasm and a larger round nucleus. In both diseases, activated PECs express de novo the marker protein CD44 [1] and upregulate or phosphorylate specific signaling molecules $[2,3]$. In RPGN, they show primarily an increased proliferation while in FSGS migration and matrix production appear to be more prominent. This observation suggests that PECs may become activated in different fashions. It will be a major challenge to characterize PEC activation in more detail to identify specific markers which can be used diagnostically as well as for mechanistic insights.

\section{Rapidly Progressing Glomerulonephritis}

RPGN summarizes a variety of glomerular pathologies. It is the most severe form of glomerular pathologies. Irreversible loss of renal function may occur within days to several months. For this reason, high-dose glucocorticoids in combination with highly effective immunosuppressants are used to treat this entity of diseases. In most cases, an inflammatory stimulus triggers the formation of aberrant accumulations of proliferating cells within Bowman's space. In antiglomerular basement membrane disease (also Goodpasture syndrome), autoantibodies binding to collagen IV of the glomerular basement membrane trigger RPGN (type I). This disease is rare, but renal insufficiency may progress very rapidly. Glomerular depo- sition of immune complexes in lupus erythematosus or postinfectious glomerulonephritis (GN) may also trigger RPGN (type II). In pauci-immune GN (type III), no antibody or complement deposition can be observed and the exact trigger for proliferating cells in Bowman's capsule remains not entirely resolved. Independently of the primary triggering event, these proliferating cells may block the tubular outlet so that urinary flow is no longer possible. As a consequence, the entire nephron degenerates and this is one of the major reasons for irreversible loss of renal function [4].

For a long time, the origin of these 'extracapillary proliferations' or 'cellular crescents' was a matter of debate and it could only recently be resolved with the novel methodology of 'cell fate tracking'. Thus, it has become possible to genetically tag and trace PECs in vivo using an inducible transgenic mouse line with transcriptional activity specifically in PECs in the glomerulus [5]. Via this approach it could be shown that proliferating cells in Bowman's space are mostly derived from PECs [1] and also (to a varying but lesser extent) podocytes $[1,6]$. This finding was confirmed in human and rodent biopsies, which also contained predominantly PECs $[2,7,8]$.

Interestingly, classical crescents could also be induced in transgenic mice when partially ablating PECs via inducible transgenic expression of diphtheria toxin [9]. In the absence of an inflammatory stimulus, surviving PECs became activated and continued to proliferate even after the original number of PECs had been restored. The cellular crescents, which formed subsequently, were indistinguishable from other inflammatory models of cellular crescent formation and even a typical periglomerular infiltrate was formed.

In summary, activated PECs are the major constituents of cellular crescents. Pharmacological inhibition of these cells may represent a major opportunity for novel therapies for this group of glomerular diseases.

\section{Focal and Segmental Glomerulosclerosis}

Glomerulosclerosis (FSGS) is the final common pathway of virtually all primary glomerular diseases, which may ultimately lead to renal insufficiency. An estimated $10 \%$ of the general population suffers from varying degrees of renal insufficiency. Because renal insufficiency is one of the most significant risk factors for cardiovascular complications (e.g. stroke or myocardial infarction), glomerulosclerosis has become a silent but major burden for health care systems.
Moeller/Smeets 
So far, most studies investigating the pathogenesis of FSGS have focused on 'primary' FSGS, i.e. glomerulosclerosis without light-microscopic evidence of any underlying glomerular pathology. However, the 'secondary' forms of FSGS, i.e. the forms where the underlying glomerular disease can be diagnosed from a renal biopsy, are much more common. In general, an injury to podocytes appears to be necessary for glomerulosclerosis, as demonstrated in podocyte-specific transgenic depletion studies [10-12] or by the identification of podocyte-specific genes in human FSGS patients $[13,14]$.

The first characteristic lesion of glomerulosclerosis is an adhesion between the glomerular tuft and Bowman's capsule, an extracapillary lesion which can be detected by standard light microscopy $[15,16]$. Dijkman et al. [17] showed in serial sections that every segmental sclerotic lesion was connected to Bowman's capsule via an adhesion. Because several earlier studies from Japan had already suggested that PECs might be involved in the formation of a sclerotic lesion, it was proposed that these adhesions could provide the entry site for PECs to invade the segment of the tuft affected by sclerosis. Again, definite proof was provided by genetic cell fate tracking in transgenic mice. Using different models of FSGS, it was shown that PECs do indeed invade segments of the glomerular tuft via adhesions [18]. Furthermore, it was shown that invading PECs were again 'activated' (i.e. they express de novo CD44) and that the PEC activation within each glomerulus was focal - mostly in proximity to a sclerotic lesion. The presence of PECs in sclerotic lesions has also been observed in human biopsies $[8,18,19]$. For a detailed description of the pathogenesis of FSGS, refer to the review article of Smeets and Moeller [20].

Several open questions remain. The first is about the role of PECs in sclerotic lesions: do PECs try to repair or replace injured podocytes or are PECs actively involved in the formation of the sclerotic lesion (i.e. a regenerative vs. degenerative function of PECs)? An important advance in this respect was the development of a specific single-chain antibody which detects specific heparan sulfate moieties of heparan sulfate proteoglycans derived from PECs (termed LKIV69) [21]. Using this antibody, it could be shown that PECs deposit significant amounts of their own but 'foreign' extracellular matrix within sclerotic segmental lesions in FSGS [18]. Furthermore, PECs could so far only be observed within sclerotic lesions and not within the histologically intact parts of the glomerular tuft $[18,22]$. These observations argue that PECs have a degenerative function in the context of glomerulosclerosis.
Secondly, activated PECs are indistinguishable in FSGS and RPGN using the currently known marker proteins. Still, functional differences remain, such as migration and matrix production versus proliferation, respectively. Future studies will have to investigate the role of the potentially diverging functions of individual signaling pathways for PEC activation in both disease entities, i.e. FSGS versus RPGN.

Third, in a recent study it was proposed that podocytes may acquire a PEC phenotype in a transgenic model of FSGS [23]. However, these results may need to be interpreted with caution since it has been shown that podocyte proteins may also be expressed in PECs [24], and in the study of Sakamoto et al. [23] a constitutively active Cre mouse was used to label podocytes. In general, aberrant labeling of cells other than podocytes (e.g. PEC) cannot be ruled out when using constitutively active Cre mice.

\section{Concluding Remarks}

Traditionally, glomerular pathologies are classified according to descriptive terms, i.e. 'proliferative' or 'sclerotic'. The emerging role of activated PEC is a common finding in proliferative and sclerotic glomerular diseases. This finding implies that common mechanisms and/or signaling pathways may be active in both groups of glomerular diseases. In a reanalysis of the literature, the tyrosine-kinase inhibitor Gleevec showed beneficial effects in a model of RPGN as well as of FSGS [25]. In a recent study by the group of Benigni, more potentially relevant signaling pathways were proposed, such as the CXCR4/ SDF-1 and renin-angiotensin systems [2]. Future studies will likely identify more candidate signaling pathways. Another major step has been taken towards the ultimate goal: to identify the essential pathways for PEC activation, which can be inhibited pharmacologically.

\section{Acknowledgements}

This work was supported by TP17 of the SFB/Transregio 57 of the German Research Foundation (DFG; to M.J.M.), the eRARE consortium Rare-G (01 GM 1208A to M.J.M.), and a grant from the DFG (Bo 3755/1 to B.S.). M.J.M. is a member of the SFB/TRR57 consortium Mechanisms of Organ Fibrosis (DFG). 


\section{References}

1 Smeets B, Uhlig S, Fuss A, Mooren F, Wetzels JF, Floege J, Moeller MJ: Tracing the origin of glomerular extracapillary lesions from parietal epithelial cells. J Am Soc Nephrol 2009; 20:2604-2615.

>2 Rizzo P, Perico N, Gagliardini E, Novelli R, Alison MR, Remuzzi G, Benigni A: Nature and mediators of parietal epithelial cell activation in glomerulonephritides of human and rat. Am J Pathol 2013;183:1769-1778.

>3 Zhang J, Pippin JW, Krofft RD, Naito S, Liu Z, Shankland SJ: Podocyte repopulation by renal progenitor cells following glucocorticoids treatment in experimental FSGS. Am J Physiol Renal Physiol 2013;304:F1375F1389.

-4 Le Hir M, Besse-Eschmann V: A novel mechanism of nephron loss in a murine model of crescentic glomerulonephritis. Kidney Int 2003;63:591-599.

$>5$ Appel D, Kershaw DB, Smeets B, Yuan G, Fuss A, Frye B, Elger M, Kriz W, Floege J, Moeller MJ: Recruitment of podocytes from glomerular parietal epithelial cells. J Am Soc Nephrol 2009;20:333-343.

6 Moeller MJ, Soofi A, Hartmann I, Le Hir M, Wiggins R, Kriz W, Holzman LB: Podocytes populate cellular crescents in a murine model of inflammatory glomerulonephritis. J Am Soc Nephrol 2004;15:61-67.

$\checkmark 7$ Thorner PS, Ho M, Eremina V, Sado Y, Quaggin S: Podocytes contribute to the formation of glomerular crescents. J Am Soc Nephrol 2008;19:495-502.

$>8$ Smeets B, Angelotti ML, Rizzo P, Dijkman H, Lazzeri E, Mooren F, Ballerini L, Parente E, Sagrinati C, Mazzinghi B, Ronconi E, Becherucci F, Benigni A, Steenbergen E, Lasagni L, Remuzzi G, Wetzels J, Romagnani P: Renal progenitor cells contribute to hyperplastic lesions of podocytopathies and crescentic glomerulonephritis. J Am Soc Nephrol 2009;20:2593-2603.
$>9$ Sicking EM, Fuss A, Uhlig S, Jirak P, Dijkman H, Wetzels J, Engel DR, Urzynicok T, Heidenreich S, Kriz W, Kurts C, Ostendorf T, Floege J, Smeets B, Moeller MJ: Subtotal ablation of parietal epithelial cells induces crescent formation. J Am Soc Nephrol 2012;23:629-640.

10 Macary G, Rossert J, Bruneval P, Mandet C, Belair MF, Houillier P, Duong Van Huyen JP: Transgenic mice expressing nitroreductase gene under the control of the podocin promoter: a new murine model of inductible glomerular injury. Virchows Arch 2009;456: 325-337.

$>11$ Matsusaka T, Xin J, Niwa S, Kobayashi K, Akatsuka A, Hashizume H, Wang QC, Pastan I, Fogo AB, Ichikawa I: Genetic engineering of glomerular sclerosis in the mouse via control of onset and severity of podocyte-specific injury. J Am Soc Nephrol 2005;16:1013-1023.

$>12$ Wharram BL, Goyal M, Wiggins JE, Sanden SK, Hussain S, Filipiak WE, Saunders TL, Dysko RC, Kohno K, Holzman LB, Wiggins RC: Podocyte depletion causes glomerulosclerosis: diphtheria toxin-induced podocyte depletion in rats expressing human diphtheria toxin receptor transgene. J Am Soc Nephrol 2005;16:2941-2952.

13 Boute N, Gribouval O, Roselli S, Benessy F, Lee H, Fuchshuber A, Dahan K, Gubler MC, Niaudet P, Antignac C: NPSH2, encoding the glomerular protein podocin, is mutated in autosomal recessive steroid-resistant nephrotic syndrome. Nat Genet 2000;24:349-354.

-14 Buscher AK, Konrad M, Nagel M, Witzke O, Kribben A, Hoyer PF, Weber S: Mutations in podocyte genes are a rare cause of primary FSGS associated with ESRD in adult patients. Clin Nephrol 2012;78:47-53.

15 Kriz W, Gretz N, Lemley KV: Progression of glomerular diseases: is the podocyte the culprit? Kidney Int 1998;54:687-697.

-16 Van Damme B, Tardanico R, Vanrenterghem Y, Desmet V: Adhesions, focal sclerosis, protein crescents, and capsular lesions in membranous nephropathy. J Pathol 1990;161:4756.

17 Dijkman H, Smeets B, van der Laak J, Steenbergen E, Wetzels J: The parietal epithelial cell is crucially involved in human idiopathic focal segmental glomerulosclerosis. Kidney Int 2005;68:1562-1572.
18 Smeets B, Kuppe C, Sicking EM, Fuss A, Jirak $P$, van Kuppevelt TH, Endlich K, Wetzels JF, Grone HJ, Floege J, Moeller MJ: Parietal epithelial cells participate in the formation of sclerotic lesions in focal segmental glomerulosclerosis. J Am Soc Nephrol 2011;22:12621274.

19 Fatima H, Moeller MJ, Smeets B, Yang HC, D’Agati VD, Alpers CE, Fogo AB: Parietal epithelial cell activation marker in early recurrence of FSGS in the transplant. Clin J Am Soc Nephrol 2012;7:1852-1858.

20 Smeets B, Moeller MJ: Parietal epithelial cells and podocytes in glomerular diseases. Semin Nephrol 2012;32:357-367.

21 Wijnhoven TJ, Lensen JF, Rops AL, van der Vlag J, Kolset SO, Bangstad HJ, Pfeffer P, van den Hoven MJ, Berden JH, van den Heuvel LP, van Kuppevelt TH: Aberrant heparan sulfate profile in the human diabetic kidney offers new clues for therapeutic glycomimetics. Am J Kidney Dis 2006;48:250-261.

22 Berger K, Schulte K, Boor P, Kuppe C, van Kuppelvelt TH, Floege J, Smeets B, Moeller MJ: The regenerative potential of parietal epithelial cells in adult mice. J Am Soc Nephrol 2014, E-pub ahead of print.

23 Sakamoto K, Ueno T, Kobayashi N, Hara S, Takashima Y, Pastan I, Matsusaka T, Nagata $\mathrm{M}$ : The direction and role of phenotypic transition between podocytes and parietal epithelial cells in focal segmental glomerulosclerosis. Am J Physiol Renal Physiol 2014;306:F98F104.

24 Guhr SS, Sachs M, Wegner A, Becker JU, Meyer TN, Kietzmann L, Schlossarek S, Carrier L, Braig M, Jat PS, Stahl RA, MeyerSchwesinger C: The expression of podocytespecific proteins in parietal epithelial cells is regulated by protein degradation. Kidney Int 2013;84:532-544.

25 Moeller MJ, Smeets B: Novel target in the treatment of RPGN: the activated parietal cell. Nephrol Dial Transplant 2013;28:489-492. 